\title{
Low-Cost Radar-Based Target Identification Prototype using an Expert System
}

\author{
David Pérez, Mónica Villaverde , Félix Moreno
}

\author{
Noemí Nogar, Félix Ezcurra and Ekaitz Aznar .
}

\section{INTRODUCTION}

Nowadays, smart and green cities concept makes people be aware about all the wasted energy in street lighting and light pollution at night.

City traffic and human security at night depend on night lighting because it is essential to prevent accidents, crimes or other kind of problems that arise in the darkness. Ming et al. [1] analyzed the relationship among population, crime and city sky glow. In order to avoid these problems, regions with high population density tend to consume large amounts of light energy. However, in many places within those regions a significant part of public lighting is wasted. Therefore, many current researches are focus on finding different solutions to reduce these massive losses.

In order to reduce energy consumption and to evolve towards better green and smart cities, renewable energy-based systems could be applied. However, the most common way for reducing the power consumption is to replace the traditional lamps by others more efficient based on LED technology. Apart of those alternatives, there is another one based on regulating the intensity of streetlamps or turning them on only when something of interest is on the road. But these alternatives can be combined each other to improve the energy saving.

Some studies focus on the streetlamp lighting technology. For example, Burgos-Payán et al. [2] made a comparison among different lighting systems and Alzubaidi and Kumar [3] developed a simulation software to investigate the efficient street lighting scheme through the use of different types of lamps. However, combinations of different alternatives are more common. Ahmed et al. [4] analyzed the effect of mounting angle of solar panels and their size to powered LED street light lamps whereas Dalla et al. [5] combined the use of solar energy and LED technology in order to design an autonomous street lighting system.

Because most of the energy in public road is wasted lighting empty spaces, the power on adjustment may be useful. This is the issue that the paper addresses. Consequently, the main goal of this work is to classify the objects that would require street lighting, as pedestrian and vehicles by creating a low cost platform for detecting and identifying these objects presented on the road. Furthermore, different methodologies are analyzed in order to create an expert system that provides the best results in terms of identification success rate.

This paper is organized as follows. In section II related works are presented. Section III describes the system hardware architecture and the procedures and methodologies followed to achieve a good classification. Section IV shows the obtained results for different algorithms. And finally, in the last section some conclusions are given.

\section{RELATED WORK}

Detecting presence on the road is not a very complicated task and there are several ways to detect movements. Liu et al. [6] used buried coils in order to detect the variation of the magnetic field produced by a metallic object. This kind of detection is good for vehicles but it is not able to detect people on the road. 
Optical methods, like systems based on videocameras [7] or laser devices [8], could be useful for detection. However, these methods are not very appropriated for a low cost application because they are computationally too complex and require expensive devices.

Another possibility is to use ultrasonic systems but, in this case, the short range of these devices makes impossible a real implementation for this application. A very similar option is to employ radar-based systems. Radar technology uses an electromagnetic wave instead of an ultrasonic wave; so long range could be achieved using radar devices.

Among all of the above mentioned alternatives, the radar technology is the selected one for this work. Radar detection is not a too complex task because the device provides a signal when some motion occurs. However, difficulties appear when a moving object has to be identified because the provided signal has to be analyzed.

There are several procedures to identify objects using radar echoes. For example, Fang et al. [9] utilized time-frequency analysis, multi-threshold detection, and Hough transform as the main signal processing methods to extract speed and shape information of vehicles whereas Alaee and Amindavar [10] applied Chirplet transform to radar signal, extracted some motion features via Zernike moments and finally made the target classification using the nearest neighbor clustering technique. Moreover, a two-stage support vector machine (SVM) classification method using Mel-Frequency Cepstrum coefficients is developed and proposed in [11]. Another alternative is proposed by Gharaibeh and Yaqot [12]. They presented a methodology based on Particle Swarm Optimization (PSO) that improved the computational speed for the nearest neighbor clustering technique. Other kind of objects are analyzed in [13] where Briones et al. developed a radar system that operates in land and air and classifies different targets using an artificial neural network trained with the Levenberg-Marquardt algorithm.

The proposed system in this paper analyses the radar echo in both the time and frequency domains in order to classify different kinds of targets using an expert system based on classification trees and correlation function. This procedure does not require very high performance devices.

\section{IMPLEMENTATION}

\section{A. System architecture}

One of the main goals of this work is to obtain a low cost platform, and for this reason a low cost radar is used. The system allows two kinds of radar devices; one used for short range applications [14] and the other one, for long range applications [15]. Both radar devices work in X-band (10.525 $\mathrm{GHz}$ ) with a continuous wave and they are based on the Doppler Effect (1), where the radial object speed is $v_{0}$, the working radar frequency is $f_{0}$ and the light speed is $c$.

Due to the fact that the Doppler signal $f^{\prime}$ is a very high frequency signal, the device gives an intermediate frequency $\Delta f$ which is lower than the first one (2).

$$
\begin{gathered}
f^{\prime}=f_{0} \cdot\left(\frac{c}{c \pm v_{0}}\right) \\
\Delta f=f_{0}-f^{\prime}
\end{gathered}
$$

This signal has a very low amplitude, offset and noise, so a conditioning stage is required. A bandwidth filter eliminates the offset and the noise and two amplification stages adapt it to a DSP (Digital Signal Processor) levels.

The core of the system is a DSP (dsPIC33FJ64MC802 device from Microchip) which uses a 16 bits modified Harvard architecture achieving up to $40 \mathrm{MIPS}$. In addition, the platform has some external communication capabilities such as an $I^{2} \mathrm{C}$ and an USB interface.

The power consumption of the whole system, including the radar device, is less than 1.6 watt when supplied with an operating voltage of 12 volts.

Fig. 1 shows the system architecture whereas Fig. 2 illustrates the real prototype.

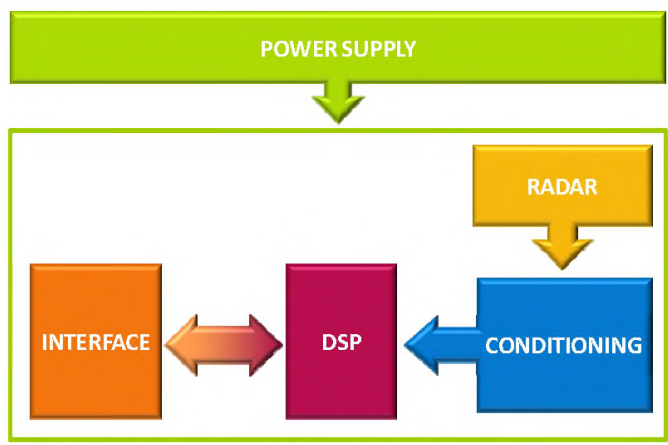

Fig. 1. Block diagram of the system.

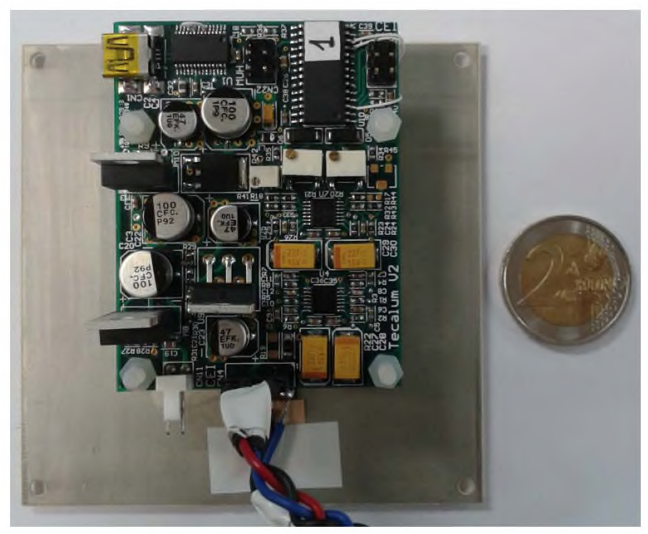

Fig. 2. Current prototype system

\section{B. Procedures and Methodologies}

The objective of this work is the lighting of the streetlamps, so technically it is not necessary to make a very accurate identification. Only three categories have been taken into account: cars, pedestrian and environments whose echoes characteristics largely depend on many factors such as volume, size, material, form, speed and trajectory. Then, it will be possible to integrate others kinds of objects in the system. 
For a correct object matching (hit), echo signals have to be processed in frequency and time domains because both domains have different and useful information. However, the system not only analyzes several frequency and time parameters by means of the extraction of some characteristics but also a correlation function is taken into account to compare real signals with some patterns stored in the system in order to find the best result (Fig. 3). In that case, patterns have to be created taking different signals of the same category and applying some kind of statistical operation.
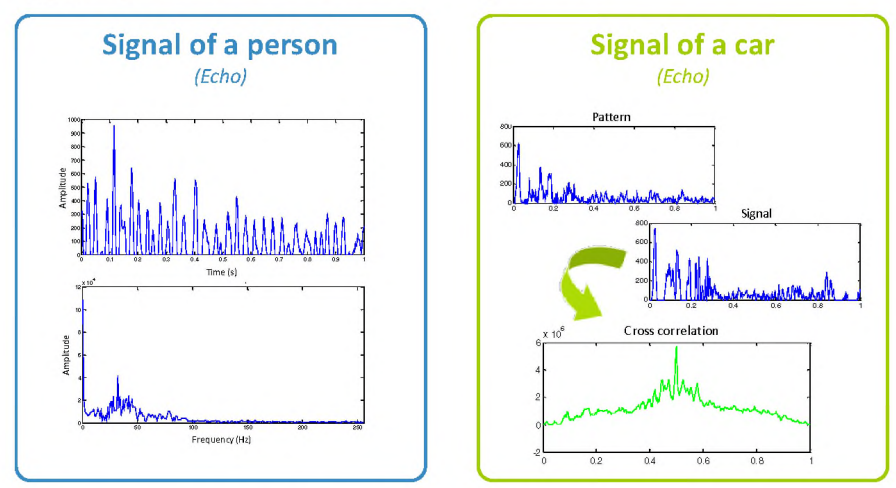

Fig. 3. Parameters extraction.

Processing all of that information it is possible to make the identification using an expert system. In this case, the expert system is based on a classification tree that uses statistical operations in order to increase the hit rate.

In machine learning, a classification tree is a predictive model defined by a training set that is composed by instances, attributes and classes [16], [17]. Each instance is an example that has several attributes and belonging to a specific class (Fig. 4). There are several statistical algorithms that can be used to create classification trees. In this work, three classes have been defined -cars, pedestrians and environments- and two classification tree algorithms have been analyzed.

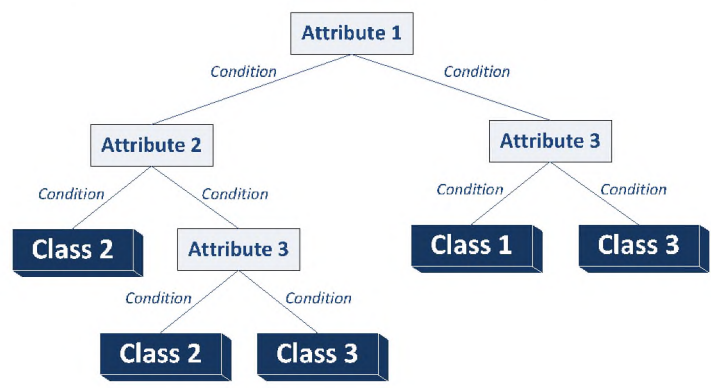

Fig. 4. Generic classification tree.

CRT (Classification and Regression Tree) [16] algorithm tries to hold the internal homogeneity among nodes while CHAID (CHi square Automatic Iteration Detection) [17] algorithm is based on selecting the attribute which splits data for giving the strongest interaction with the chosen class by using the chi square test. The most widely used algorithm in this paper is CRT, but sometimes CHAID gives better results.

In any case, if some attribute or a number of instances change, the designed classification tree will be different.
Therefore, it is very important to define a good training set in order to get an optimal knowledge source.

\section{Verification}

In order to test the system and to verify results a new tool is developed. This tool stores an image and the signal that represents the echo and analyzes results to find the best identification algorithm, for this reason a webcam is needed.

Taking into account that it is possible to execute different identification algorithms in the DSP at the same time, this external tool allows to compare all the DSP algorithms results observing the real object that appear in the image.

That tool is encoded in LabView and it is divided in different modules to manage the control of the application, to select the configuration parameters, to specify the webcam and to change detection parameters.

\section{RESULTS}

In this section the proposed methodology is shown. For this purpose many experiments have been carried out. First of all, the correlation function and classification trees were tested theoretically using simulations to determine if it possible to use them for classifying different signals. After that, both methods were implemented to test the system running in different real situations. Using the external tool designed for verification it was possible to discuss the validation of the different algorithms.

\section{A. Theoretical results}

In this stage the identification is made using external software therefore the dsPIC is only in charge of detecting objects and obtaining signals. To capture signals, the radar is located at street level and detected signals are stored in order to analyze them with external software. Fig. 5 shows a real location for the device.
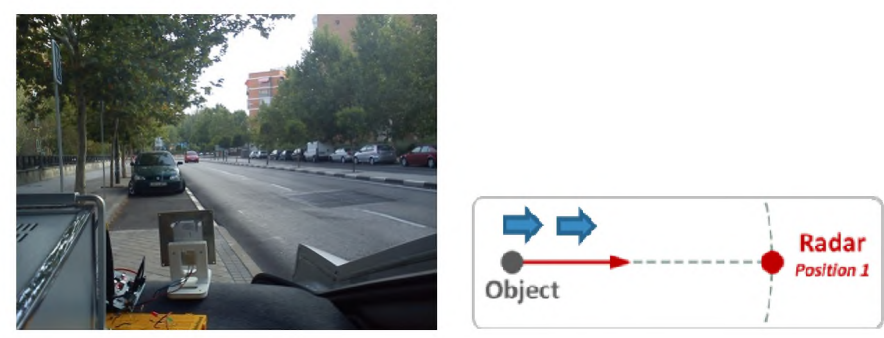

Fig. 5. Radar location at street level.

In order to compare different methods of identification, the collected data is analyzed based on correlation function and based on classification trees.

Using Matlab it is possible to study how the correlation function could be useful to make identifications. In that case it is necessary to analyze the cross correlation between the radar signal -when an object is detected- and all the patterns that represent each class. To make different classification trees based on different statistical procedures, the statistical package SPSS [18] is used. 
Moreover, focusing on correlation function, the best results are obtained by analyzing the amplitudes of the peaks belonging to the central samples of the cross correlation (Fig. 6 ). The radar signal is correlated with all of the stored patterns and all of these peaks are analyzed to determine the class. The higher amplitude implies the greater similarity.

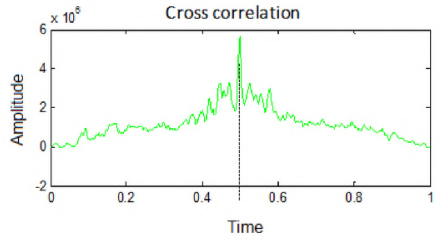

Fig. 6. Cross correlation analysis.

On the other hand, a classification tree gives the estimated probability. It means that different statistical methodologies have different results for the same attributes, the same classes and the same number of instances. Accordingly, the best method is the one that gives the higher probability. In this study and for the selected examples, the CRT is the classification tree that offers the best results. Fig. 7 illustrates the theoretical results obtained in this stage. It demonstrates that classification tree gives higher hit rates than correlation function, especially for pedestrian identification. In any case, environment hit rates are around 50\% due to few environment instances are defined in the used training set.

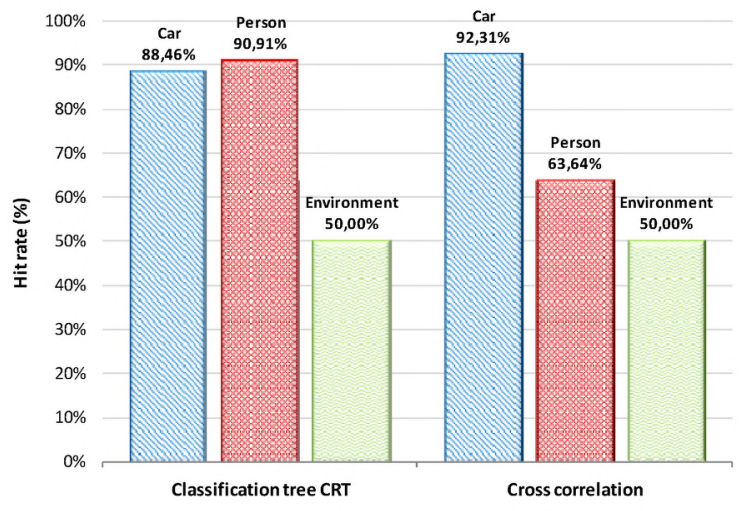

Fig. 7. Theoretical results.

\section{B. Practical results}

To make these tests, it is necessary to change to a more realistic place. Consequently, the radar is located at four or five meters over the street level (Fig. 8).
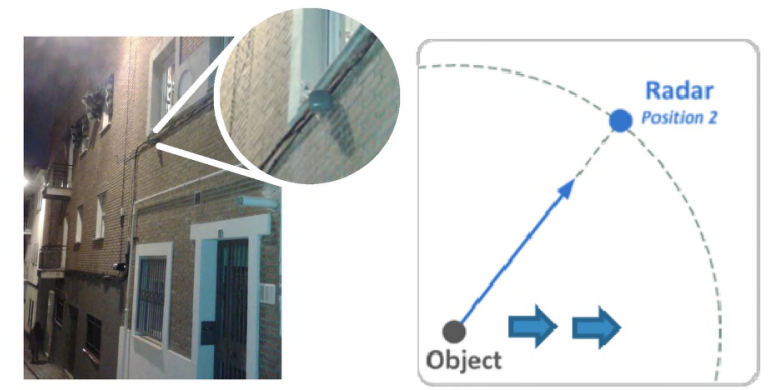

Fig. 8. Radar location over the street level.
Moreover, in this stage, the system is in charge of detecting, capturing and identifying. Also, different algorithms have been implemented in order to provide different alternatives to find the methodology that provides the best results. 1, 2 and 4 algorithms use the signal captured in the previous stage (with radar al street level) whereas the rest of algorithms (3, 5 y 6$)$ use signals taken at four or five meters over the street level. Furthermore, there are differences among the implementation of each algorithm. On the one hand, algorithm 5 uses a CHAID-based classification tree and on the other hand, 1, 2, 3, 4 and 6 algorithms use a CRT-based classification. However, algorithm 2 includes a reinforcement of frequency attribute and algorithm 4 takes into account the results of the correlation function.

It is also important to point out that in practical results I and II, environment signals are not included because not enough information was available from the environment.

The earliest tests were carried out using signals captured in theoretical stage but placing the radar at the new location. Fig. 9 illustrates those results.

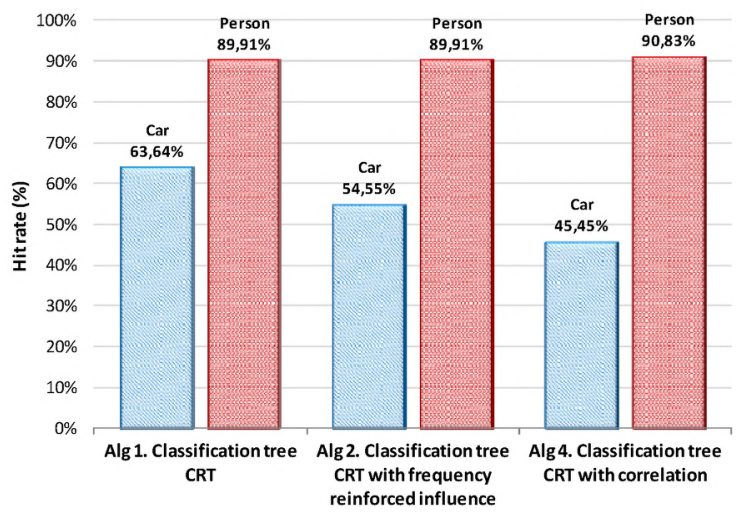

Fig. 9. Practical results I.

Despite of the fact that the correlation function presents good theoretical results, its results are worse when it is combined with classification tree algorithms. Furthermore, the classification tree shows worse results comparing them to its own theoretical results. Consequently, new signals were needed considering the new radar location in order to improve the hit rate probability. Fig. 10 shows those results.

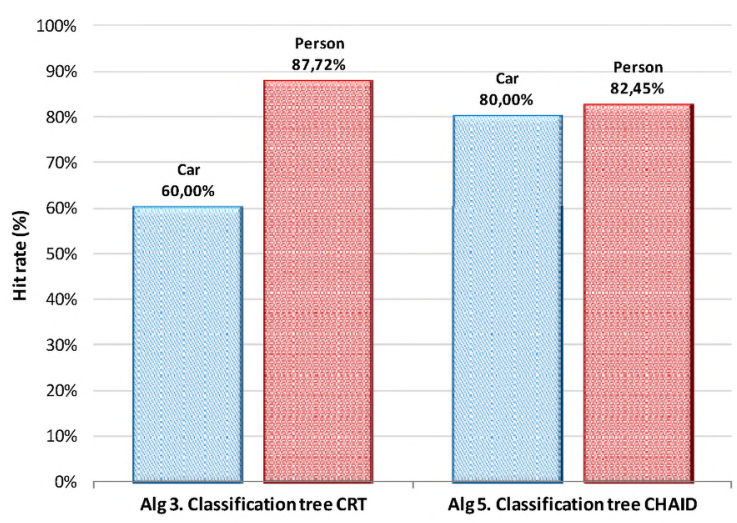

Fig. 10. Practical results II. 
In the last test, the correlation function is not taken into account because results are not good enough and a new kind of classification tree is studied (CHAID).

The following step was to analyze the enviroment hit rate and to carry it out, more environment signals were taken. The next test is focused on improving results when forecast conditions are not fair enough.

Results change due to several environment signals are included in the analysis (Fig. 11). These new signals represent rain weather conditions.

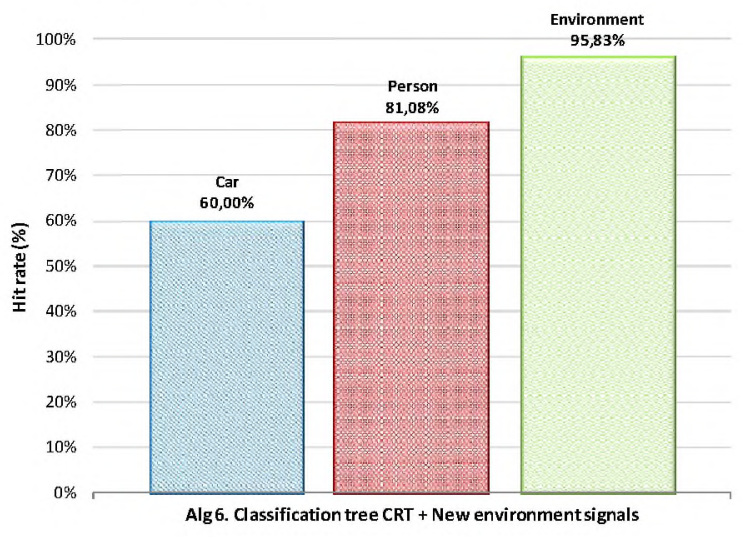

Fig. 11. Practical results III.

A very important issue is the fact that the system does not require high accuracy in the identification process. Therefore, a new kind of probability can be defined by taking into account that the system works properly if the streetlight turns on only when either a person or a car is moving on the street. This new item is called the effective hit rate. Therefore, the effective hit rate is defined as the probability of lighting when either a car or a person is detected by the system. It means that the system does not necessarily have to differentiate between people or cars.

Fig. 12 shows results from that point of view (effective hit rate). Only two of those algorithms have been selected because they are the two ones providing better results (CHAID-based classification tree and CTR-based classification tree with new environment signals).

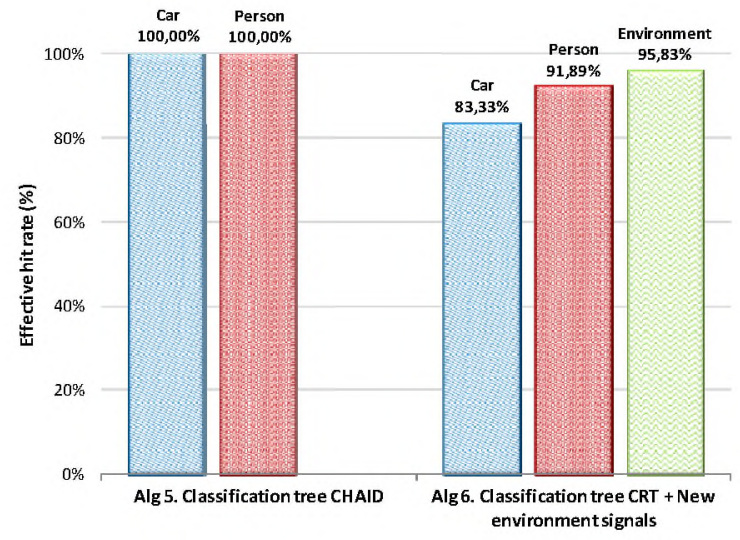

Fig. 12. Practical results IV. Effective hit rate.
TABLE I. REAL Hit RATE. Theoretical REsUlts

\begin{tabular}{|l|c|c|}
\cline { 2 - 3 } \multicolumn{1}{c|}{} & \multicolumn{2}{c|}{ REAL HIT RATE: Theoretical results } \\
\cline { 2 - 3 } \multicolumn{1}{c|}{} & CRT & Correlation Function \\
\hline Car & $88,46 \%$ & $92,31 \%$ \\
\hline Person & $90,91 \%$ & $63,64 \%$ \\
\hline Environment & $50,00 \%$ & $50,00 \%$ \\
\hline
\end{tabular}

TABLE II. Real Hit Rate. Practical Restlts

\begin{tabular}{|l|c|c|c|l|l|l|}
\hline \multicolumn{1}{c|}{} & \multicolumn{6}{c|}{ REAL HIT RATE: Practical results } \\
\cline { 2 - 7 } \multicolumn{1}{c|}{} & \multicolumn{3}{|c|}{ Signals taken at street level } & \multicolumn{3}{|l|}{ Signals taken at 4-5 meters } \\
\cline { 2 - 7 } \multicolumn{1}{c|}{ Alg.1 } & Alg.2 & Alg.4 & Alg.3 & Alg.5 & Alg.6 \\
\hline Car & $63,64 \%$ & $54,55 \%$ & $45,45 \%$ & $60,00 \%$ & $80,00 \%$ & $60,00 \%$ \\
\hline Person & $89,91 \%$ & $89,91 \%$ & $90,83 \%$ & $87,72 \%$ & $82,45 \%$ & $81,08 \%$ \\
\hline Environment & - & - & - & - & - & $95,83 \%$ \\
\hline
\end{tabular}

TABLE III. EFfective Hit Rate. Practical Results

\begin{tabular}{|l|c|c|}
\cline { 2 - 3 } \multicolumn{1}{c|}{} & \multicolumn{2}{c|}{ EFFECTIVE HIT RATE: Practical results } \\
\cline { 2 - 3 } \multicolumn{1}{c|}{} & Alg.5 & Alg.6 \\
\hline Car & $100,00 \%$ & $83,33 \%$ \\
\hline Person & $100,00 \%$ & $91,89 \%$ \\
\hline Environment & - & $95,83 \%$ \\
\hline
\end{tabular}

TABLE IV. COMPARATIVE OF DIFFERENT IDENTIFICATION PROCEDURES

\begin{tabular}{|l|c|}
\hline \multicolumn{2}{|c|}{ AVERAGE HIT RATE } \\
\hline Classification tree (Alg.5) & $81,22 \%$ \\
\hline Particle Swarm Optimization & $73,13 \%$ \\
\hline Two-stage SVM procedure & $98,10 \%$ \\
\hline
\end{tabular}

\section{CONCLUSIONS}

The most innovative contribution of this paper is related to its application field, since it uses a low-cost system based on radar detection instead of more expensive alternatives, such as videocameras or laser devices, for lighting streetlamps management. The proposed prototype, together with artificial inteligence methodologies, is able to control the public street lighting saving a huge amount of energy. The paper proposes the use of classification trees as an appropriated mechanism to achieve it, since they are suitable to classify radar signals in order to identify different kind of objects. As results demonstrate, the system achieves high hit rates and its results, in terms of effective hit rate, are nearly to the maximum values (Table III).

An important factor is that radar hit rate is very variable depending on the device location and weather conditions. Consequently, the system location dependence is a very important issue because the signal provided by the radar, and therefore the identification results, is strongly linked to the device position. As shown in Table II, an appropriated location for radar device provides higher hit rates. For this reason, 1, 2 
and 4 algorithms results that use the initial signals taken at street level (Fig.9) are worse than 3 and 5 algorithms results which use new signals taking into account the height of the radar location (Fig. 10). Moreover, correlation function was ignored in the last tests because its results were deteriorated due to the fact that signals are more similar among differents classes when the radar is located at higher positions.

The dependence on weather conditions is another important issue, and for this reason this work includes the algorithm 6 (Fig.11). This algorithm demonstrates that it is advisable -and even mandatory in some cases- to take several radar signals considering usual wheather conditions in the area. Thus, as a conclusion, if the number of environment and weather signals increases, the hit rate increases as well.

In spite of the radar location and weather influency, the obtained results reach considerably higher hit rates. In fact, these hit rates outperform the values obtained by using PSO algorithm [12]. However, the two-stage SVM prodedure [11] results are better but they did not work in a real scenario and their radar echo signals presented no interference due to the fact that it worked in a controlled environment without the presence of multiple targets (Table IV).

In order to avoid depencences and to increase the system hit rates, a more complex artificial intelligence methologies could be included. If the system is able to adquire knowledge, the clasification will be better, its identifications will be more accurate and its location and weather dependences will be reduced. Another way to improve classification hit rates is to consider more than one identification for each target, developing a complex system with several devices working together. In the future, learning capabilities will be included in the system for improving its results and also a collaborative network will be created for deciding the target class jointly.

\section{ACKNOWLEDGMENT}

This work is being developed under the call INNPACTO2011 (ITP-2011-1977-920000), currently financed by the Spanish Ministry of Economy and Competitiveness (previously Ministry of Science and Innovation) within the National Plan for Scientific Research, Development and Technological Innovation, 2008-2011. 